\title{
Adherencia al tratamiento inmunosupresor en el paciente adulto con trasplante renal
}

\author{
María Isabel Durán Muñoz, Teresa Lope Andrea, María Rosario del Pino Jurado, María Cristina Chicharro \\ Chicharro, Elisa Matilla Villar
}

Enfermeras. Servicio de Nefrología del Hospital Clínico San Carlos. Madrid

\section{Sr. Director:}

En España durante el año 2010 se hicieron 2225, de los cuales 420 se realizaron en Madrid. (www.ont.es) El número de trasplantes renales realizados en este hospital desde sus inicios en el año 1980, son 1740.

El cumplimiento adecuado del tratamiento inmunosupresor es fundamental en este tipo de pacientes, ya que la no adherencia al tratamiento puede ser la causa de la pérdida del injerto renal; se ha comprobado lo importante que es seguir correctamente dicho tratamiento junto con hábitos de vida saludables para que mejore también la supervivencia del paciente $\mathrm{e}^{(1,2,3,4,4,5,6,7,8,9)}$.

La adherencia al tratamiento puede ser una causa modificable, donde tiene un papel importante la enfermería, interviniendo de manera activa en la educación sanitaria del paciente trasplantado $(1,2,3,4,5,6,7,8)$, de ahí, que nos planteemos conocer el grado de adherencia que tienen nuestros pacientes con trasplante renal.

Es difícil detectar los receptores de trasplante renal que no son adherentes al tratamiento inmunosupresor, pero a su vez es importante identificarlos para poder actuar con prontitud y en consecuencia, pues en muchas ocasiones, se puede sospechar de un mal cumplimiento
Correspondencia:

María Isabel Durán Muñoz

Servicio de Nefrología

Hospital Clínico San Carlos

Calle Profesor Martín Lagos s/n. 28040. Madrid

e-mail: iduran72@gmail.com del tratamiento, sin afirmarlo hasta que realmente lo reconoce el paciente ${ }^{(1,2,3,4,5,6,7,8)}$.

La adherencia al tratamiento se debe al adecuado cumplimiento terapéutico inmunosupresor, que engloba dos conceptos: el cumplimiento de dosis y forma de administración y, la persistencia en la duración del tratamiento prescrito $(1,2,3,4,5,6,7)$.

A pesar de la importancia de la adherencia a la medicación inmunosupresora en la supervivencia del trasplante renal, existen pocos estudios en España y la mayoría de los estudios realizados son estadounidenses.

Como en otros estudios de adherencia a medicamentos en pacientes con trastornos crónicos, los factores más destacados que influyen en la adherencia se clasifican en: el propio paciente, el medicamento, la enfermedad, y la calidad de la interacción entre el paciente y el sistema sanitario.

Nos planteamos como objetivo, conocer el grado de adherencia a la terapia inmunosupresora en pacientes adultos, mayores de 18 años, con trasplante renal funcionante en nuestro hospital.

Como objetivos secundarios de nuestro estudio nos propusimos:

- Identificar factores que influyen en la adherencia al tratamiento inmunosupresor (Socio-demográficos, medicamentos, información sanitaria).

- Describir las barreras que encuentran los pacientes con trasplante renal para adherirse al tratamiento inmunosupresor. 


\section{Material y métodos}

\section{Diseño y ámbito \\ Estudio descriptivo a través de un corte transver- sal en pacientes adultos, mayores de 18 años, con injerto renal funcionante, que acuden a la consulta de trasplante renal, de ámbito hospitalario del Hospital Clínico San Carlos.}

La selección de pacientes se llevó a cabo de manera consecutiva y desde el comienzo del estudio, a todos los pacientes adultos, mayores de 18 años, con injerto renal funcionante, que acudían a la consulta de trasplante, eran autosuficientes en el manejo de su tratamiento y deseaban participar en dicho estudio. Se entregó hoja informativa sobre el estudio a realizar y se pidió consentimiento informado.

\section{Recogida de datos}

La recogida de datos consistió en la cumplimentación de un cuestionario auto-administrado, anónimo y no identificativo que se les facilitó a los pacientes y que conllevaba menos de 15-20 minutos en rellenarlo. La entrega de los cuestionarios se hizo en sobre cerrado y se recogieron en un buzón que dispusimos para ello.

\section{Herramienta de estudio}

Como herramienta de estudio se elaboró un cuestionario de 43 preguntas repartidas de la siguiente manera:

- 10 preguntas referentes a datos socio-demográficos del paciente.

- 7 preguntas referentes a la toma de medicación en general, tanto inmunosupresora como otras medicaciones para otras patologías asociadas.

- 6 preguntas referentes a la medicación inmunosupresora específica del trasplante.

- 4 preguntas referentes a la información recibida por el personal sanitario.

- En 4 preguntas se utilizó la escala validada para medir la adherencia al tratamiento inmunosupresor, escala ITAS (inmunosuppessant therapy adherence scale) (Anexo I), que estima la frecuencia con la que los pacientes no han sido adherentes a la medicación en los últimos tres meses. En los estudios de validación, el ITAS ha demostrado estar asociado a la frecuencia de rechazo del injerto y a las concentraciones séricas de inmunosupresores. La escala original consta de 4 ítems con cuatro posibles respuestas. Los valores que toman van desde un mínimo de 0 a un máximo de 12 puntos, siendo 12 el nivel máximo de adherencia y 0 el de no adherencia.

- En 12 preguntas se utilizó la escala validada para identificar barreras a la toma de medicación inmunosupresora en pacientes con trasplante renal, escala ITBS (inmunosuppessant therapy barrier scale) (Anexo II). La escala original consta de 13 ítems con 5 respuestas tipo Likert (que evalúan intensidad o frecuencia), que a su vez se divide en dos subescalas, una considerada como barreras incontrolables porque evalúa actitudes respecto a las dificultades para la adherencia, como algo impuesto y externo al paciente y otra que se considera como barreras controlables porque dependen propiamente del paciente. Hemos decidido suprimir el ítems 13 de la escala original por considerarlo no aplicable en España al hacer referencia al coste de la medicación para el paciente. La escala resultante consta de 12 ítems, de los cuales, los 8 primeros hacen referencia a la subescala de barreras incontrolables y los 4 ítems restantes hacen referencia a la subescala de barreras controlables. Los valores que toman la escala conjunta van de 12 a 60, de 8 a 40 en la subescala de barreras incontrolables y de 4 a 20 en la de barreras controlables, significando los valores mínimos una menor presencia de barreras.

\section{Criterios de inclusión}

Pacientes con trasplante renal funcionante, adultos, mayores de 18 años, que acuden a la consulta de trasplante renal de ámbito hospitalario y son autosuficientes en el manejo de su tratamiento.

\section{Criterios de exclusión}

Se excluyeron del estudio aquellos pacientes que no quisieron participar libremente, no firmaron el consentimiento informado y los que necesitaban ayuda o no eran autónomos en el manejo de su medicación, por no ser ellos los responsables directos de la no adherencia.

\section{Tamaño muestral}

Para conseguir una precisión del 4,5\% en la estimación de una proporción mediante un intervalo de confianza asintótico Normal con corrección para poblaciones finitas al $95 \%$ bilateral, asumiendo que la proporción esperada es del $80,0 \%$ y que el tamaño total de la población era de 817, sería necesario incluir 222 pacientes en el estudio. En función del registro de 
trasplantes renales de la consulta de enfermería, el número de sujetos potenciales candidatos a participar en el estudio sería de 150 al mes. Se estimó que la mitad de esta muestra no cumpliría los criterios de inclusión y/o serían visitas consecutivas, por lo tanto fue necesario un periodo de estudio de unos cuatro meses (Junio-Septiembre 2011) para reclutar el tamaño muestral necesario.

\section{Variables de estudio}

- Variables Dependientes

- Adherencia al tratamiento: Se utilizó la escala ITAS $^{\text {[anexo I] }}$.

- Barreras de adherencia percibidas: Se utilizó la escala ITBS ${ }^{[a n e x o}$ II].

\section{- Variables Independientes}

- Factores socio-demográficos: Sexo, edad, nivel de estudios, situación laboral, situación de convivencia

- Medicación: tipo de medicación inmunosupresora.

- Educación sanitaria recibida por parte de los profesionales de la salud.

\section{Análisis estadístico:}

Se diseñó una base de datos en Access 2003 en la que se recogió la información de las variables del cuestionario.

\section{Análisis descriptivo}

Las variables cualitativas se presentan con su distribución de frecuencias. Las variables cuantitativas se resumen en su media y desviación estándar (DE). Las variables cuantitativas que no siguen una distribución normal se resumen con la mediana y el rango intercuartílico (RIC). Se calculó la frecuencia de adherencia y de los factores que conllevan el incumplimiento junto a sus IC al $95 \%$.

\section{Análisis univariado}

Se evaluó la asociación entre variables cualitativas con el test de $\chi 2$ o prueba exacta de Fisher, en caso de que más de un $25 \%$ de los esperados fueron menores de 5 . En el caso de variables ordinales se contrastó la hipótesis de tendencia ordinal de proporciones. Las comparaciones de medias entre dos grupos independientes se realizaron mediante la prueba $T$ de Student para grupos independientes. En el caso de variables que no se distribuían de manera normal se utilizó el test no paramétrico de la mediana.

\section{Análisis multivariado}

Se ajustó un modelo de regresión logística, con el objeto de evaluar la asociación de aquellas variables independientes del estudio que se relacionan con cada una de las variables dependientes dicotómicas (adherencia y causa de incumplimiento). Se incluyeron en el análisis las variables que en el análisis univariado presenten una $p<0,15$ y/o clínicamente relevantes. Se presentan los odds ratio ajustados junto con los intervalos de confianza al $95 \%$.

En todos los contrastes de hipótesis se rechazó la hipótesis nula con un error de tipo I o error menor a 0.05 .

El paquete informático que se utilizó para el análisis fue el SPSS 15.0.

\section{Consideraciones éticas:}

Protección de los datos:

En ningún caso se han incluido en las bases de datos, dato alguno que pudiera directa o indirectamente identificar a ningún sujeto de modo individualizado. Con ello, se respetan las normas internacionales de protección de datos, así como la legislación española vigente. Los investigadores responsables son garantía de la seguridad de las bases de datos, que no serán utilizadas para otro fin que el señalado en el apartado de objetivos específicos.

\section{Consentimiento informado}

Se administró la hoja de consentimiento informado a los pacientes, previo a la realización del estudio, respetando las normas de la declaración de Helsinki. En él se explicaban los objetivos y procedimientos del estudio y se aseguraba la confidencialidad de los datos. Se recogió convenientemente firmada.

Informe favorable del Comité Ético de Investigación Clínica.

\section{Resultados}

De 209 pacientes de los que se recibió el cuestionario, la edad media es de 55,2 ( $\pm 12,15)$ (media \pm D.E.) de un rango comprendido entre $21-80$ años; de los que el $61,8 \%$ son hombres y el $38,2 \%$ son mujeres. El $38,2 \%$ de estos pacientes tienen un nivel de estudios secundarios, la situación de convivencia más frecuente es: con pareja y más familia un $40,6 \%$, seguido de vivir 
con pareja y sin familia con un $30,4 \%$, lo que nos lleva a deducir que el $71 \%$ de nuestros pacientes conviven con pareja. En cuanto a la situación laboral, la condición de jubilado es la más frecuente con un $42,0 \%$ y la menos frecuente es la de estudiante con un 1,9\%.

En cuanto a la medicación inmunosupresora que toman, el medicamento inmunosupresor, sólo o combinado, más utilizado entre nuestros pacientes es el MYFORTIC ${ }^{\circledR}$, con un $65,5 \%$, seguido del PROGRAF ${ }^{\circledR}$, con un $56,8 \%$. El $46,7 \%$ refieren que toman dos inmunosupresores.

En cuanto a la toma de esta medicación, la manera de recordar la toma en un $61,7 \%$, es de memoria y si tuvieran que evitar una de las tomas, preferirían la de la noche un $57,2 \%$, porque según refieren les permite organizar mejor su ocio y relaciones personales $(41,0 \%)$ y porque se adapta mejor a su vida familiar (30,1\%).

En cuanto a los efectos secundarios de los inmunosupresores, el 54,6\% de los pacientes encuestados, refiere no tener ninguno; siendo los problemas dermatológicos con un $28,5 \%$, los más referidos, seguido de problemas musculo-esqueléticos con un $28,0 \%$ y gastrointestinales con un $25,5 \%$.

Nuestros pacientes, creen que la información recibida por parte de los profesionales sanitarios sobre los beneficios de la medicación es la adecuada en un $87,9 \%$. En cambio, sobre la información de los efectos adversos, un $20,5 \%$, opina que es insuficiente y sólo un $73,7 \%$ creen que es la adecuada. La información sobre las horas a las que deben tomar la medicación, el $88,8 \%$ opina que es fácil de entender y el $96,6 \%$ refiere que siguen las instrucciones que le dieron los profesionales sanitarios.

La adherencia al tratamiento inmunosupresor medida con la escala ITAS (escala de 0-12), fue respondida por 201 pacientes con una puntuación media de 11,7( \pm 1,03 ). El valor máximo de 12 significa una adherencia al tratamiento inmunosupresor muy buena.

El 88,1\% (I.C.95\%:83,3-92,8) de nuestros pacientes son adherentes al tratamiento inmunosupresor $(n=177)$.

La media de edad de los adherentes es mayor que la de los no adherentes $(56,3( \pm 11,8)$ vs $47,7( \pm 11,8)$; $p=0,001$ ).
También es de destacar que de los pacientes con estudios universitarios, el $20 \%$ no son adherentes $(p=$ $0,044)$ y de los que están en situación laboral en activo, el $22,4 \%(p=0,010)$. No se ha encontrado significación con el resto de variables.

El análisis de la escala de barreras (ITBS), se ha hecho en conjunto y por separado de las dos subescalas (Figuras 1 y 2 ). Los valores mínimos significan una menor presencia de barreras.

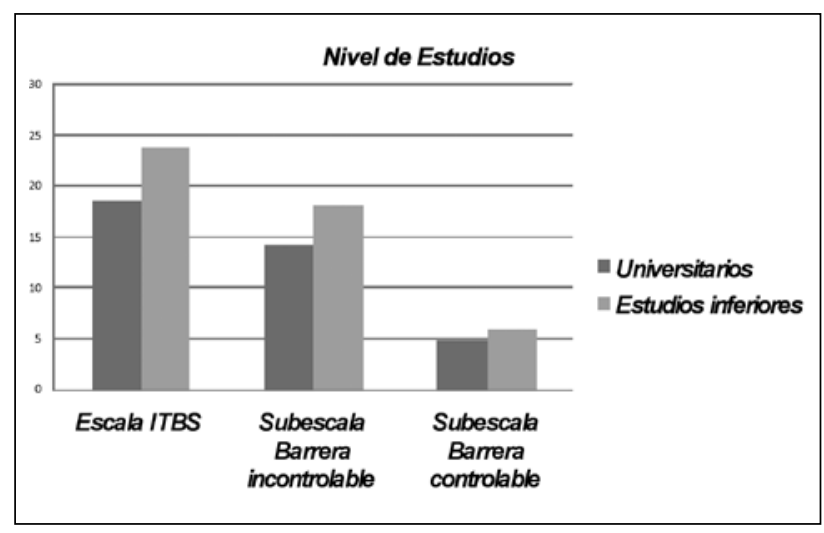

Figura 1

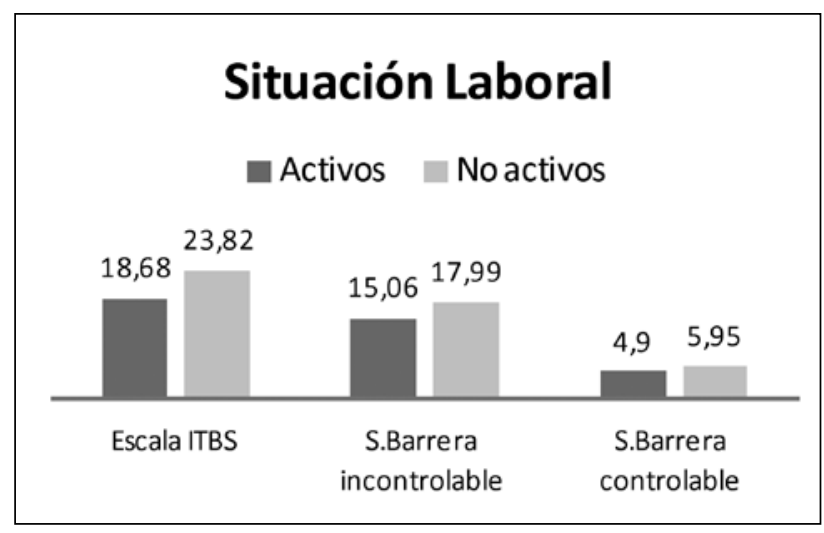

Figura 2

La percepción de barreras a la toma de medicación inmunosupresora en pacientes con trasplante renal, medidas con la escala ITBS (escala de 12-60), fue respondida por 176 pacientes con una puntuación media de $22,7( \pm 7,7)$. No hay diferencias por edad y sexo. En cuanto al nivel de estudios, los pacientes que tienen estudios universitarios, presentan puntuaciones más bajas $(18,68( \pm 4,96))$ que el resto $(23,82( \pm 7,94))(p=0,000)$. Los pacientes que 
se encuentran en activo, la media de puntuación es de $19,93( \pm 5,31)$ frente al resto, cuya media es de 23,73( \pm $8,23)(p=0,004)$. Los pacientes adherentes presentan una puntuación de $22,07( \pm 7,36)$ frente a los no adherentes $26,21( \pm 8,35)(p=0,024)$. No se ha encontrado significación con el resto de variables.

La subescala de barreras incontrolables (puntuación de 8-40), fue respondida por 181 pacientes con una puntuación media de $17,2( \pm 5,57)$. Los $\leq$ de 45 años presentan una puntuación más baja $14,93( \pm 3,94)$ frente a los de $>46$ años que presentan una media de $17,62( \pm 5,77)(p=0,015)$. No hay diferencias en el sexo ni en la situación de convivencia; en cuanto al nivel de estudios que tienen los pacientes, los que poseen estudios universitarios presentan valores más bajos $14,14( \pm 3,65)$ frente al resto $18,07( \pm 5,71)$ $(p=0,000)$. Los pacientes que se encuentran en activo presentan puntuaciones menores $15,06( \pm 4,06)$ frente a los no activos $17,99( \pm 5,87)(p=0,000)$. Existe una tendencia a la significación estadística en los pacientes adherentes cuya puntuación es $19,20( \pm 5,65)$ frente a los no adherentes $16,82( \pm 5,44)(p=0,024)$. No se ha encontrado significación con el resto de variables.

La subescala de barreras controlables (puntuación de 4-20), fue respondida por 195 pacientes con una puntuación media de $5,67( \pm 2,82)$. No hay diferencias por edad, sexo, ni situación de convivencia; en cuanto al nivel de estudios que tienen los pacientes, los que poseen estudios universitarios presentan valores más bajos 4,83 $\pm 1,96$ frente al resto $5,87( \pm 2,96)(p=0,008)$. Los pacientes que se encuentran en activo presentan puntuaciones menores $4,9 \pm 1,86$ frente a los no activos $5,95( \pm 3,05)(p=0,005)$. Los pacientes adherentes presentan una puntuación media de $5,47( \pm 2,67)$ frente a los no adherentes $6,86( \pm 3,29)(p=0,027)$. No se ha encontrado significación con el resto de variables.

\section{Conclusiones}

Estamos ante un grupo de pacientes cuya media de edad son 55 años y mayoritariamente están jubilados y conviven en pareja.

El nivel de no adherencia obtenido ha sido bajo, y el perfil de los pacientes menos adherentes son los pacientes más jóvenes, con estudios universitarios, y los que están en activo.
Las barreras de adherencia percibidas son bajas, siendo los pacientes con estudios universitarios y que se encuentran en activo, los que presentan menor percepción de estas barreras.

No se han encontrado diferencias significativas entre las dos subescalas de barreras (barreras controlables e incontrolables).

Los pacientes adherentes perciben menos barreras de adherencia que los no adherentes.

Consideramos necesario tomar medidas de mejora en cuanto a la información sanitaria sobre los efectos adversos de la medicación inmunosupresora, ya que nuestros pacientes la consideran insuficiente.
Recibido: 10 Febrero 2012
Revisado: 10 Marzo 2012
Modificado: 5 Septiembre 2012
Aceptado: 10 septiembre 2012

\section{Bibliografía}

1. Chisholm MA, Lance CE, Williamson GM, Mulloy LL. Development and validation of the immunosuppressant therapy adherence instrument (ITAS). Patient Educ Couns. 2005; 59:13-20.

2. Chisholm MA, Lance $C E$, Williamson GM, Mulloy LL. Development and validation of an immunosuppressant therapy adherence barrier instrument. Nephrol Dial Transplant. 2005; 20:181-188.

3. Chisholm MA, Lance CE, Mulloy LL. Patient factors associated with adherence to immunosuppressant therapy in renal transplant recipients. Am J Health Syst Pharm.2005 Sep 1; 62(17): 1775-1781.

4. Chisholm-Burns MA, Spivey CA, Wilks SE. Social support and immunosuppressant therapy adherence among adult renal transplant recipients. Clin Transplant. 2010 May-Jun; 24(3):312-320.

5. Wilks SE, Spivey CA, Chisholm-Burns MA. Psychometric re-evaluation of the immunosuppressant therapy adherence scale among solid-organ trans- 
plant recipients. Journal of Evaluation in Clinical Practice. 2010 Feb; 16(1):64-68.

6. Fernandez Lucas M, López Sanchez S, Miranda B, Matesanz R. Adhesión al tratamiento en pacientes receptores de trasplante renal. Estudio multicéntrico español. Nefrología.1998; XVIII: 316-325.

7. Nogués Solán X, Sorli Redó ML, Villar García J. Instrumentos de medida de adherencia al tratamiento. Anales de Medicina Interna. 2007;24(3):138-141.

8. Barquero Ruano RM, Checa Barambio E, Rodriguez Peña J. Mala adherencia al tratamiento inmunosupresor de un paciente trasplantado renal. Rev Soc Esp Enferm Nefr.2010 0ct-Dic; 13(4):267-269.

9. Butler JA, Roderick P, Mullee M, Mason JC, Peverer RC, Frecuency and impact of nonadherence to immunosuppressants after renal transplantation: a systematic review.Transplantation.2004 Mar;77(5):769-776.

\section{ANEXO I}

\section{Escala de adherencia al tratamiento inmunosu- presor (ITAS)}

1. En los últimos 3 meses ¿con qué frecuencia olvido tomar la medicación inmunosupresora?

2. En los últimos 3 meses ¿con que frecuencia descuidó tomar los inmunosupresores

3. En los últimos 3 meses ¿con que frecuencia dejó de tomar sus medicamentos inmunosupresores porque se encontraba peor?

4. En los últimos 3 meses ¿con que frecuencia dejó de tomar sus medicamentos inmunosupresores por los motivos que fuese?

\section{Las posibles respuestas de son:}
A. Nunca
B. A veces (menos del $20 \%$ de las veces)
C. Con frecuencia(entre el $20 \%$ y el $50 \%$ de las veces)
D. Más de la mitad de las veces

\section{ANEXO II \\ Escala de barrera para la adherencia al tratamiento inmunosupresor (ITBS)}

1. Tengo que tomar los medicamentos inmunosupresores demasiadas veces al día

2. Tengo que tomar demasiadas cápsulas o pastillas de los medicamentos inmunosupresores en una sola vez

3. No podría asegurar que los medicamentos inmunosupresores me estén ayudando

4. Me salto dosis de los medicamentos inmunosupresores cuando voy de viaje

5. Me salto dosis de los medicamentos inmunosupresores cuando estoy deprimido

6. No estoy seguro de cómo debo tomar los medicamentos inmunosupresores

7. No entiendo cuando tengo que tomar los medicamentos inmunosupresores

8. Con frecuencia se me acaban o me quedo sin medicamentos inmunosupresores

9. Me resulta difícil acordarme de tomar los medicamentos inmunosupresores

10. Me salto una dosis, cuando creo que tengo efectos secundarios de los medicamentos inmunosupresores

11. A veces me salto una dosis de los medicamentos inmunosupresores cuando me siento bien

12. Me salto dosis de los medicamentos inmunosupresores cuando salgo de la rutina

\section{Las posibles respuestas son:}
A. Completamente de acuerdo
B. De acuerdo
C. Normal
D. En desacuerdo
E. Completamente en desacuerdo 